\title{
Discharge by Noon: The Time Has Come for More Times to be the Right Time
}

\author{
Emily R Kane, MD, MS ${ }^{1,2 *}$; Evan Fieldston, MD, MBA, MSHP1,2
}

${ }^{1}$ Children's Hospital of Philadelphia, Philadelphia, Pennsylvania; ${ }^{2}$ University of Pennsylvania Perelman School of Medicine, Philadelphia, Pennsylvania.

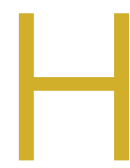

ospitalists have become well versed in campaigns championing safe, efficient, and timely discharges, as well as in the pragmatic challenges of achieving them. Successfully discharging a patient from the hospital requires synchronizing several elements; as a result, improvement efforts focus on promoting shared mental models and team identification of early discharges. The urgency for timely discharges, much like (and unlike') hotel check-out times, becomes increasingly relevant when hospitals are functioning at or beyond full capacity. As inpatient medical care grows increasingly more specialized, promoting high-quality discharges theoretically allows for not only more beds, but also that the right bed is available for the right patient at the right time. In addition, financial realities in terms of reimbursement and the high cost of adding capacity imply that hospitals need to maximize throughput from the beds they already have. For these reasons, hospital administrators and operational leaders have focused on early discharges as a goal-and have often used discharge before noon (DCBN) as the metric to measure performance.

In this issue of the Journal of Hospital Medicine, Destino et al. reported that it is possible to achieve a higher percentage of early discharges, which allowed for decompression of post-anesthesia care and emergency areas without a measurable negative impact on patient or family satisfaction or length of stay (LOS). ${ }^{2}$ The improvement they report is remarkable. However, it will be important for them to report back, as quality improvement projects often revert to prior state unless the processes are reinforced and embedded in hospital culture. In addition, what goes unreported in Destino et al. are the unmeasured and unanticipated outcomes related to focusing on a single, laudable goal. This study and others have yet to confirm that systems have enough resiliency to improve discharge timeliness without diverting resources from other aspects of care. ${ }^{3}$ In other words, can inpatient teams do everything at the same time without sacrificing quality; ie, improve discharge timeliness, accept and admit new patients faster, respond to deteriorating patients, spend enough time with patients and families to meet their needs (and validated survey expectations), and in educational settings, meet the learning needs of trainees? ${ }^{4}$ This may prove to be true if implementation techniques are individualized to hospitals, services, and units and are incorporated into existing workflows, minimizing extraneous "asks" on already overtaxed

${ }^{*}$ Corresponding Author: Emily Kane, MD, MS; E-mail: KaneE1@email.chop. edu Telephone: 215-590-1000.

Received: October 30, 2018; Accepted: November 1, 2018

() 2019 Society of Hospital Medicine DOI 10.12788/jhm.3122 providers. Evidence to support this would go a long way in engaging stakeholders to prioritize quality discharges.

In this issue, too, James, et al. ask the question "if DCBN is a good indicator of shorter LOS or is DCBN an arbitrary indicator. ${ }^{\prime \prime}$ The answer may be yes, no, both, maybe, and it depends. Certainly, no pathophysiological reasons exist for a certain time of day to be the "right" time for discharge. The key question for hospitalists and health systems leaders is whether setting time goals leads clinicians to delay discharges of medically and logistically ready patients in the afternoon or evening, particularly if the metric is linked to monetary performance incentives. This is also likely a matter of degrees, ie, set the DCBN goal at $80 \%-100 \%$ and gaming is much more likely; set the goal at $20 \%-30 \%$ and this might reflect a realistic range and be less likely to incentivize gaming. Notably, the hospital in the James study did not have a DCBN goal. It would be interesting to see what would happen in that hospital or another hospital before and after implementing a DCBN goal—and further assess a dose-response curve. Another approach would be to perform qualitative analysis of readiness for discharge via chart reviews and determine if patients could have left in the afternoon or evening but might have been delayed to buff up the performance on the DCBN metric.

James et al. additionally demonstrate differences for medical and surgical patients, underscoring that a DCBN goal is unlikely to yield the same results in different patient cohorts or settings. The authors note several workflow reasons for this variation, but other considerations are regularity of timelines for recovery being different for surgical patients, role of elective admissions scheduled in advance, and the potential use of conditional orders (ie, orders entered before dawn that nurses can activate as patients meet criteria).

What both studies highlight is that although morning discharges can help with patient flow, hospitalists and hospital leaders need to be mindful and seek more information before implementing DCBN programs. One strategy that can promote efficient discharge regardless of the position of the sun in the sky, account for variation in patient populations and individual patients, and mitigate the potential for gaming the system is to strive toward measuring time from medical readiness to the time of discharge. Although some institutions have had success with this work, it remains challenging to implement this across all patient populations. Criteria for medical readiness need to be agreed upon and validated, and then a real-time way of identifying when criteria are met needs to be developed. In this regard, hospitals may have to invest individually or collectively to build such systems, but the benefit would be to enable and promote performance of timely dis- 
charge for all patients at all times of day.

Much as we have adopted cultural changes over the years to raise awareness regarding patient safety such as nosocomial infections and hand hygiene, an emphasis on high-quality discharges too needs to become integral to hospital practices to sustain performance and any associated metrics. As to what to measure? A validated "medical readiness to discharge" may be the gold standard but may be difficult to attain. Until then, carefully constructed approaches to prioritizing early discharges through proactive planning, shared mental models, interdisciplinary teamwork, and appropriate incentives to those who do it well could yield the results we want as hospitalists, as patients, and as families.

Disclosures: Dr. Kane and Dr. Fieldston have nothing to disclose.

\section{References}

1. lantorno S, Fieldston E. Hospitals are not hotels: high-quality discharges occur around the clock. JAMA Pediatr. 2013;167(7):596-597. doi: 10.1001/jamapediatrics.2013.2252.

2. Destino L BD, Acuna $C$, Asch S, Platchek T. Improving patient flow: analysis of an initiative to improve early discharge. J Hosp Med. 2019;14(1):22-27. doi: 10.12788/JHM.3133.

3. Lorch SA, Millman AM, Zhang $X$, et.al. Impact of admission-day crowding on the length of stay of pediatric hospitalizations. Pediatrics. 2008;121(4):e718-e730. doi: 10.1542/peds.2007-1280.

4. Haferbecker D, Fakeye O, Medina SP, Fieldston ES. Perceptions of educational experience and inpatient workload among pediatric residents. Hosp Pediatri. 2013;3(3):276-284. doi: 10.1542/hpeds.2012-0068.

5. James H, Steiner MJ, Holmes GM, Stephens JR. The association of discharge before noon and length of stay in hospitalized pediatric patients. $J$ Hosp Med. 2019:14(1):28-32. doi: 10.12788/jhm.3111.

6. White $C M$, Statile $A M$, White $D L$, et al. Using quality improvement to optimize paediatric discharge efficiency. BMJ Qual Saf. 2014;23(5):428-436. doi: 10.1136/bmjgs-2013-002556. 